\title{
NORMALIZED LAPLACIAN SPECTRUM OF SOME $Q$-CORONAS OF TWO REGULAR GRAPHS
}

\author{
Arpita Das and Pratima Panigrahi \\ Department of Mathematics \\ Indian Institute of Technology Kharagpur \\ India, 721302 \\ e-mail: arpita.das1201@gmail.com \\ pratima@maths.iitkgp.ernet.in
}

\begin{abstract}
In this paper we determine the normalized Laplacian spectrum of the $Q$ vertex corona, $Q$-edge corona, $Q$-vertex neighborhood corona, and $Q$-edge neighborhood corona of a connected regular graph with an arbitrary regular graph in terms of normalized Laplacian eigenvalues of the original graphs. Moreover, applying these results we find some non-regular normalized Laplacian co-spectral graphs.

Keywords: Normalized Laplacian matrix, $Q$-vertex corona, $Q$-edge corona, $Q$-vertex neighborhood corona, $Q$-edge neighborhood corona, Kronecker product, Hadamard product.
\end{abstract}

2010 Mathematics Subject Classification: 05C50; 47A75.

\section{REFERENCES}

[1] A. Banerjee and J. Jost, On the spectrum of the normalized graph Laplacian, Linear Algebra Appl. 428 (2008) 3015-3022. doi:10.1016/j.laa.2008.01.029

[2] S. Butler and J. Grout, A construction of cospectral graphs for the normalized Laplacian, Electr. J. Combin. 18 (2011) 231pp.

[3] F.R.K. Chung, Spectral Graph Theory (CBMS. Reg. Conf. Ser. Math. 92, AMS, providence, RI, 1997).

[4] D. Cvetković, P. Rowlinson and S. Simić, An Introduction to the Theory of Graph Spectra (Cambridge University Press, 2009).

[5] A. Das and P. Panigrahi, Normalized Laplacian spectrum of different type of coronas of two regular graphs, Kragujevac J. Math. 41 (2017) 57-69. 
[6] A. Das and P. Panigrahi, Normalized Laplacian spectrum of some subdivisioncoronas of two regular graphs, Linear and Multilinear Algebra 65 (2017) 962-972. doi:10.1080/03081087.2016.1217976

[7] A. Das and P. Panigrahi, Normalized Laplacian spectrum of some R-coronas of two regular graphs, South. Asian Bull. Math. 42 (2018) 833-844.

[8] C. Godsil and G. Royle, Algebraic Graph Theory (Springer, New York, 2001).

[9] F. Harary, Graph Theory (Addison-Wesley, Reading, PA, 1969).

[10] R.A. Horn and C.R. Johnson, Topics in Matrix Analysis (Cambridge University Press, Cambridge, 1991).

[11] J. Huang and S.C. Li, On the normalised Laplacian spectrum, degree-Kirchhoff index and spanning trees of graphs, Bull. Aust. Math. Soc. 91 (2015) 353-367. doi:10.1017/S0004972715000027

[12] H.H. Li and J.S. Li, A note on the normalized Laplacian spectra, Taiwanese J. Math. 15 (2011) 129-139.

[13] Q. Liu, J.B. Liu and J. Cao, Further results on resistance distance and Kirchhoff index in electric networks (Discrete Dynamics in Nature and Society, 2016). doi:10.1155/2016/4682527

[14] C. McLeman and E. McNicholas, Spectra of coronae, Linear Algebra Appl. 435 (2011) 998-1007.

doi:10.1016/j.laa.2011.02.007

[15] E.R. Vandam and W.H. Haemers, Which graphs are determined by their spectrum?, Linear Algebra Appl. 373 (2003) 241-272.

doi:10.1016/S0024-3795(03)00483-X

Received 28 January 2019

Revised 19 September 2019

Accepted 11 October 2020 